\title{
On the distance spectrum of minimal cages and associated distance biregular graphs
}

\author{
Aditi Howlader and Pratima Panigrahi \\ Department of Mathematics, Indian Institute of Technology Kharagpur, India \\ e-mail: aditihowlader21@gmail.com, pratima@maths.iitkgp.ernet.in
}

September 14, 2021

\begin{abstract}
A $(k, g)$-cage is a $k$-regular simple graph of girth $g$ with minimum possible number of vertices. In this paper, $(k, g)$-cages which are Moore graphs are referred as minimal $(k, g)$-cages. A simple connected graph is called distance regular(DR) if all its vertices have the same intersection array. A bipartite graph is called distance biregu$\operatorname{lar}(\mathrm{DBR})$ if all the vertices of the same partite set admit the same intersection array. It is known that minimal $(k, g)$-cages are DR graphs and their subdivisions are DBR graphs. In this paper, for minimal $(k, g)$-cages we give a formula for distance spectral radius in terms of $k$ and $g$, and also determine polynomials of degree $\left\lfloor\frac{g}{2}\right\rfloor$, which is the diameter of the graph. This polynomial gives all distance eigenvalues when the variable is substituted by adjacency eigenvalues. We show that a minimal $(k, g)$-cage of diameter $d$ has $d+1$ distinct distance eigenvalues, and this partially answers a problem posed in [5]. We prove that every DBR graph is a 2-partitioned transmission regular graph and then give a formula for its distance spectral radius. By this formula we obtain the distance spectral radius of subdivision of minimal $(k, g)$-cages. Finally we determine the full distance spectrum of subdivision of some minimal $(k, g)$-cages.
\end{abstract}

Keywords: Distance spectrum; Distance regular graph; Distance biregular graph; Minimal $(k, g)$-cage; Subdivision graph; $k$-partitioned transmission regular graph.

Subclass: $05 C 12,05 C 50$ 


\section{Introduction and Preliminaries}

In this article, by a graph we mean a finite, simple, connected and undirected graph. Let $G=(V, E)$ be a graph with vertex set $V(G)=\left\{v_{1}, v_{2}, \ldots, v_{n}\right\}$ and edge set $E(G)=\left\{e_{1}, e_{2}, \ldots, e_{m}\right\}$. The adjacency matrix $A(G)$ of $G$ is an $n \times n$ matrix with $(i, j)^{\text {th }}$ entry 1 or 0 according as $v_{i}$ is adjacent to $v_{j}$ or not. The incidence matrix $R(G)$ of $G$ is an $n \times m$ matrix whose $(i, j)^{t h}$ entry is 1 or 0 according as vertex $v_{i}$ is an end vertex of edge $e_{j}$ or not. The distance matrix $D(G)$ of $G$ is an $n \times n$ matrix whose $(i, j)^{t h}$ entry is the distance (length of the shortest path) between the vertices $v_{i}$ and $v_{j}$. The eigenvalues of $A(G)$ (respectively $D(G)$ ) are called eigenvalues (respectively distance eigenvalues or D-eigenvalues) of $G$. The set of all eigenvalues (respectively distance eigenvalues) of $G$ is called the spectrum (respectively distance spectrum or D-spectrum) of $G$. If $\lambda_{1}, \lambda_{2}, \ldots, \lambda_{p}$ are distinct eigenvalues (respectively distance eigenvalues) of $G$ with respective multiplicities $m_{1}, m_{2}, \ldots, m_{p}$ then the spectrum (respectively distance spectrum) of $G$ is denoted by $\left\{\lambda_{1}^{\left(m_{1}\right)}, \lambda_{2}^{\left(m_{2}\right)}, \ldots, \lambda_{p}^{\left(m_{p}\right)}\right\}$. The largest eigenvalue of $D(G)$ is called the distance spectral radius of $G$.

The distance matrix of a graph gives several structural information of the graph. Thus the computation of the distance matrix and its characteristic polynomial is much more intense problem. Graham and Pollack [13] introduced distance matrix of a graph and established a relationship between the number of negative eigenvalues of this matrix and addressed a problem in data communication systems. The distance matrix and distance spectrum of a graph has numerous applications to chemistry [9] and other branches of science and engineering. For some recent results on the characteristic polynomials of the distance matrices and distance spectra of graphs, one may refer [2, 3, 15].

For any graph $G$ of diameter $d$, and a vertex $u \in V(G), G_{i}(u)$ denotes the set of all vertices in $G$ of distance $i$ from $u, i=0,1, \ldots, d$. A connected graph $G$ is called distance regular (in short DR) if it is regular and for any two vertices $x, y \in G$ at distance $i$, there are precisely $c_{i}$ neighbors of $y$ in $G_{i-1}(x)$ and $b_{i}$ neighbors of $y$ in $G_{i+1}(x), 0 \leq i \leq d$, where $c_{0}$ and $b_{d}$ are undefined. The sequence $\left\{b_{0}, b_{1}, \ldots, b_{d-1} ; c_{1}, c_{2}, \ldots, c_{d}\right\}$ is called the intersection array of a distance regular graph $G$. For $i=0,1, \ldots, d$, the numbers $c_{i}, b_{i}$, and $a_{i}$, where $a_{i}=l-b_{i}-c_{i}$ and $l$ is the degree of regularity of $G$, are called the $i n$ tersection numbers of $G$. Biggs [6] introduced distance regular (DR) graphs. For results on DR graphs and their link with other combinatorial structures one may refer [8,19]. Every DR-graph of diameter $d$ has exactly $d+1$ distinct adjacency eigenvalues and at most $d+1$ distinct D-eigenvalue [5]. The 
authors in [1] characterized some DR graphs with diameter three and four having exactly three distinct distance eigenvalues.

For an $n$-vertex graph $G$ with diameter $d$, the $i^{\text {th }}$ distance matrix $A_{i}$, $i=1,2, \ldots, d$, of $G$ is an $n \times n$ matrix whose rows and columns are indexed by vertices of $G$ and $(j, m)^{t h}$ entry is 1 or 0 according as distance between $j^{t h}$ and $m^{\text {th }}$ vertices is $i$ or not. Thus the distance matrix $D$ of graph $G$ can be written as

$$
D=A_{1}+2 A_{2}+\cdots+d A_{d}
$$

The adjacency matrix $A$ of a distance regular graph $G$ with diameter $d$ and its $i^{\text {th }}$ distance matrices satisfy the following recurrence relation [8].

$$
A A_{i}=c_{i+1} A_{i+1}+a_{i} A_{i}+b_{i-1} A_{i-1}, \quad A_{0}=I, \quad A_{1}=A, \quad i=0,1,2, \ldots, d
$$

Applying equation (2) we get that the $i^{t h}$ distance matrix $A_{i}$ of a distance regular graph $G$ with diameter $d$ can be expressed as a polynomial (of degree i) of its adjacency matrix $A, i=1,2, \ldots, d$. Then from (1), the distance matrix $D$ can also be written as a polynomial of $A$, say $D=p(A)$, of degree $d$. Thus for every eigenvalue $\lambda$ of $A, p(\lambda)$ is a distance eigenvalue of graph G.

A connected graph $G$ is called distance-biregular ( $D B R$ ) graph if it is bipartite and all vertices in the same partite set have the same intersection array. We denote the bi-partition of a DBR graph as $\left(V_{1}, V_{2}\right)$. The intersection arrays for vertices in $V_{1}$ and $V_{2}$ are $\left\{r, e_{1}, \ldots, e_{d_{1}-1} ; 1, f_{2}, \ldots, f_{d_{1}}\right\}$ and $\left\{s, g_{1}, \ldots, g_{d_{2}-1} ; 1, h_{2}, \ldots, h_{d_{2}}\right\}$ respectively, where $r$ is the degree of vertices in $V_{1}, s$ is the degree of vertices in $V_{2}, d_{1}=\max \left\{d(x, y): x \in V_{1}, y \in V(G)\right\}$ and $d_{2}=\max \left\{d(x, y): x \in V_{2}, y \in V(G)\right\}$. The diameter $d^{\prime}$ of $G$ is of course $\max \left(d_{1}, d_{2}\right)$. For any $u \in V_{1}$ and $v \in V_{2}$ we take $l_{i}=\left|G_{i}(u)\right|$ and $l_{i}^{\prime}=\left|G_{i}(v)\right|$, $i=0, \ldots, d^{\prime}$. We note that $l_{d^{\prime}-1} \neq 0$ and $l_{d^{\prime}-1}^{\prime} \neq 0$ though one of $l_{d^{\prime}}$ and $l_{d^{\prime}}^{\prime}$ may be zero.

Some elementary relations on the intersection arrays of a DBR graph are given below.

Lemma 1.1. ( [12]) For a DBR graph, the following relations hold true: $l_{0}=1, l_{i+1} f_{i+1}=l_{i} e_{i}, l_{0}^{\prime}=1$, and $l_{i+1}^{\prime} h_{i+1}=l_{i}^{\prime} g_{i}$.

For any graph $G$ and a vertex $v$ in it, the transmission $\operatorname{Tr}_{G}(v)$ of $v$ is the sum of distances from $v$ to all other vertices in $G$. A connected graph $G$ is called $p$-transmission regular if $\operatorname{Tr}_{G}(v)$ is $p$ for all the vertices $v$ in $G$. It is known [8] that for any vertex $u$ in a DR graph $G, G_{i}(u)$ has a constant number of vertices, say $k_{i}, i=0,1, \ldots, d$. Also $k_{i}$ satisfies the relations 
$k_{0}=1, k_{1}=l, k_{i+1} c_{i+1}=k_{i} b_{i}$ for $i=0,1, \ldots, d-1$. Thus any DR graph is a $p$-transmission regular graph, where $p=\sum_{i=0}^{d} i k_{i}$. We note that the distance spectral radius of every $p$-transmission regular graph is equal to $p$.

Definition 1.1. 7] Suppose $A$ is a real symmetric matrix whose rows and columns are indexed by elements in $X=\{1,2, \ldots, n\}$. Consider the block representation of $A$ with respect to the partition $\left\{X_{1}, X_{2}, \ldots, X_{m}\right\}$ of $X$ as $A=\left(\begin{array}{cccc}A_{11} & A_{12} & \cdots & A_{1 m} \\ A_{21} & A_{22} & \cdots & A_{2 m} \\ \cdots & \cdots & \cdots & \cdots \\ A_{m 1} & A_{m 2} & \cdots & A_{m m}\end{array}\right)$, where each $A_{i j}$ denotes the sub-matrix (block) of $A$ formed by rows indexed in $X_{i}$ and the columns indexed in $X_{j}$. Let $q_{i j}$ be the average row sum of $A_{i j}$. Then the matrix $Q=\left(q_{i j}\right)$ is called a quotient matrix of $A$. For each block $A_{i j}$, if the row sum is constant then the partition is called equitable.

Lemma 1.2. ( [7]) Let $Q$ be a quotient matrix of a real symmetric matrix $A$ corresponding to an equitable partition. Then the spectrum of $A$ contains the spectrum of $Q$.

Lemma 1.3. ( 4]) If $Q$ is a quotient matrix of a real symmetric matrix $A$ corresponding to an equitable partition, then the largest eigenvalue of $A$ is equal to the largest eigenvalue of $Q$.

A connected graph $G$ is called a $t$-partitioned transmission regular graph if there exists a partition $\bigcup_{i=1}^{t} U_{i}$ (called a $t$-partition) of the vertex set of $G$ such that for any $i, j$ (not necessarily distinct) in $\{1,2, \ldots, t\}$ and for any vertex $x \in U_{i}, q_{i j}=\sum_{y \in U_{j}} d(x, y)$ is a constant, where $d(x, y)$ is the distance between $x$ and $y$ in the graph $G$. For a $t$-partitioned transmission regular graph $G,\left\{U_{i}: i=1,2, \ldots, t\right\}$ is an equitable partition of $D(G)$. Therefore the quotient matrix of $D(G)$ with respect to this partition is $Q^{D}=\left[q_{i j}\right]_{t \times t}$, and so by Lemma 1.3 the distance spectral radius of $G$ is the largest eigenvalue of $Q^{D}$.

For positive integers $k$ and $g$, a $(k, g)$-cage is a $k$-regular simple graph of girth $g$ on minimum possible number, say $n(k, g)$, of vertices. An well known [6] lower bound for $n(k, g)$ is as given below.

$n(k, g) \geq n_{0}(k, g)= \begin{cases}1+k+k(k-1)+\cdots+k(k-1)^{d-2}+k(k-1)^{d-1}, & \text { if } g \text { is odd } \\ 1+k+k(k-1)+\cdots+k(k-1)^{d-2}+(k-1)^{d-1}, & \text { if } g \text { is even, }\end{cases}$

where $d=\left\lfloor\frac{g}{2}\right\rfloor$ is the diameter of the $(k, g)$-cage. A $(k, g)$-cage for which equality holds in the above bound is called a Moore graph or a minimal $(k, g)$-cage 
The Lemma below gives information about the possible minimal $(k, g)$ cages.

Lemma 1.4. ( [11]) There exists a Moore graph (or a minimal $(k, g)$-cage) of degree $k$ and girth $g$ if and only if

(i) $k=2$ and $g>3$, cycles;

(ii) $g=3$ and $k>2$, complete graphs;

(iii) $g=4$ and $k>2$, complete bipartite graphs;

(iv) $g=5$ and:

$k=2$, the 5 -cycle,

$k=3$, the Petersen graph,

$k=7$, the Hoffman-Singleton graph,and possibly $k=57$;

(v) $g=6,8$, or 12 , and there exists a symmetric generalized $n$-gon of order $k-1$.

It is known [6] that every minimal $(k, g)$-cage is a DR graph with intersection array $\{k, k-1, \ldots, k-1, k-1 ; 1,1, \ldots, 1, k\}$ if $g$ is even, and $\{k, k-1, \ldots, k-1, k-1 ; 1,1, \ldots, 1,1\}$ if $g$ is odd. So the intersection number $a_{i}=0$ for all minimal $(k, g)$-cages, $i=0,1, \ldots, d$. The subdivision graph $S(G)$ of the graph $G$ is obtained from $G$ by inserting a new vertex of degree 2 on each edge of $G$.

The result below gives adjacency spectrum of minimal $(k, g)$-cages.

Lemma 1.5. ( [6]) Let $G$ be a $(k, g)$-cage with diameter $d$ and $n$ vertices.

(i) If $g=2 d$ then the $d+1$ distinct eigenvalues of $G$ are $\lambda=k,-k, 2 \sqrt{k-1} \cos \frac{\pi j}{d}$, $j=1,2, \ldots, d-1$, with multiplicity $m_{\lambda}=\frac{n k}{g}\left[\frac{4 h-\lambda^{2}}{k^{2}-\lambda^{2}}\right], h=k-1,|\lambda| \neq k$.

(ii) If $g=2 d+1$ then the $d+1$ distinct eigenvalues of $G$ are $\lambda=k, 2 \sqrt{k-1} \cos a_{j}$, $j=1,2, \ldots, d$, where $a_{1}, \ldots, a_{d}$ are the distinct solutions in the interval $0<a<\pi$ of the equation $\sqrt{k-1} \sin (d+1) a+\sin d a=0$ with multiplicity of an eigenvalue $\lambda$ is given by $m_{\lambda}=\frac{n k}{g}\left[\frac{4 h-\lambda^{2}}{(k-\lambda)(f+\lambda)}\right], h=k-1, f=k+\frac{k-2}{g}$.

In this paper, for minimal $(k, g)$-cages we give a formula for distance spectral radius in terms of $k$ and $g$, and also determine polynomials of degree $\left\lfloor\frac{g}{2}\right\rfloor$ which give all distance eigenvalues when the variable is substituted by adjacency eigenvalues. The authors in [5] proved that every DR graph with diameter $d$ has at the most $d+1$ distinct D-eigenvalues and then asked for characterization of DR graphs which will have exactly $d+1$ distinct D-eigenvalues. We show that all minimal $(k, g)$-cages of diameter $d$ have $d+1$ distinct distance eigenvalues. In [12] it is proved that every distanceregularized graph is either DR or DBR. The authors in [16] proved that subdivision of a minimal $(k, g)$-cage is a DBR graph. We prove that every DBR-graph is a 2-partitioned transmission regular graph and then give a 
formula for its distance spectral radius. By this formula we determine distance spectral radius of subdivision of minimal $(k, g)$-cages. We also find D-spectrum of subdivision of minimal $(3,5)$-cages, minimal $(3,6)$-cages, and minimal $(k, g)$-cages for $g=3$ and 4 with any values of $k \geq 3$.

Next we state some known results which will be used in the sequel.

Definition 1.2. [17] Let $A=\left(a_{i j}\right)$ be an $m \times n$ matrix and $B=\left(b_{i j}\right)$ be a $p \times q$ matrix then the Kronecker product of $A$ and $B$, denoted by $A \otimes B$, is defined as the $m p \times n q$ partition matrix $\left(a_{i j} B\right)$. The product of two kronecker products gives another kronecker product: $(M \otimes P)(N \otimes Q)=M N \otimes P Q$, in case where each multiplication makes sense.

Recall that for any graph $G$, its line graph $L(G)$ is the graph whose vertex set is $E(G)$ and two vertices are adjacent if the corresponding edges in $G$ share a common end vertex.

Lemma 1.6. ( [10]) Let $G$ be an $r$-regular graph with adjacency matrix $A$, incidence matrix $R$, and line graph $L(G)$. Then $R R^{T}=A+r I, R^{T} R=$ $A(L(G))+2 I, J R=2 J=R^{T} J$ and $J R^{T}=r J=R J$, where $I$ is the identity matrix and $J$ is the all-one matrix of appropriate order.

Lemma 1.7. ( [10]) Let $\mathrm{G}$ be an $r$-regular graph with $p$ vertices, $q$ edges, and eigenvalues $\left\{r, \lambda_{2}, \ldots, \lambda_{p}\right\}$. Then spectrum of $L(G)$ is $\left\{2 r-2, \lambda_{2}+\right.$ $\left.r-2, \ldots, \lambda_{p}+r-2,-2^{(q-p)}\right\}$. Also, $Z$ is an eigenvector corresponding to the eigenvalue -2 if and only if $R Z=0$, where $R$ is the incidence matrix of $G$.

\section{Distance spectrum of minimal cages}

Here we give a formula for distance spectral radius of minimal $(k, g)$-cages.

Theorem 2.1. The distance spectral radius of a minimal $(k, g)$-cage, $k \geq 3$, is

$$
\lambda_{1}=\left\{\begin{array}{ll}
\frac{k\left\{1-(k-1)^{d}\right\}}{(2-k)^{2}}-\frac{2 d(k-1)^{d}}{(2-k)}, & g \text { even } \\
\frac{k\left\{1-(k-1)^{d}\right\}}{(2-k)^{2}}-\frac{d k(k-1)^{d}}{(2-k)}, & g \text { odd }
\end{array}, \text { where } d=\left\lfloor\frac{g}{2}\right\rfloor .\right.
$$

Proof. Since a minimal $(k, g)$-cage is a DR graph which is also a $p$-transmission regular graph, the distance spectral radius of this graph is the transmission $p$ of any vertex $x$ in it. From the intersection array of the minimal $(k, g)$-cage we get, $p=\sum_{y \in G} d(x, y)=k+2 k(k-1)+3 k(k-1)^{2}+\cdots+(d-1) k(k-$ $1)^{d-2}+d c(k-1)^{d-1}$, where $c=1$ for $g$ even and $c=k$ for $g$ odd. For $g$ even, $p=k+2 k(k-1)+3 k(k-1)^{2}+\cdots+(d-1) k(k-1)^{d-2}+d(k-1)^{d-1}=$ 
$k+2 k(k-1)+3 k(k-1)^{2}+\cdots+(d-1) k(k-1)^{d-2}+d(k-k+1)(k-$ $1)^{d-1}=k\left[1+2(k-1)+\cdots+d(k-1)^{d-1}\right]-d(k-1)^{d}=k S-d(k-1)^{d}$, where $S=1+2(k-1)+\cdots+d(k-1)^{d-1}$. Then we get $S-(k-1) S=$ $\left[1+(k-1)+\cdots+(k-1)^{d-1}\right]-d(k-1)^{d}$. So $S=\frac{1-(k-1)^{d}}{(2-k)^{2}}-\frac{d(k-1)^{d}}{(2-k)}$ and we get the result in this case. If $g$ is odd then $p=k S$, and hence the result.

The next lemma will be useful to prove some important results of this paper.

Lemma 2.1. For integers $i$ and $j, i, j=0,1,2, \ldots, d$, consider the recurrence

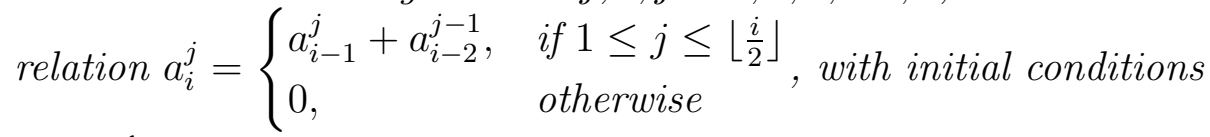
$a_{i}^{0}=\left\{\begin{array}{ll}k-1, & i=1, \ldots, d \\ k, & i=0\end{array}\right.$. Then we get,

(i) for $j>0, a_{2 j+b}^{j}= \begin{cases}k, & \text { if } b=0 \\ k+a_{2 j-1}^{j-1}+a_{2 j}^{j-1}+\cdots+a_{2 j+b-2}^{j-1}, & \text { if } b>0,\end{cases}$

(ii) $a_{i}^{j}=g_{i}^{j} k-h_{i}^{j}$ for $1 \leq j<\left\lfloor\frac{i}{2}\right\rfloor, i=4, \ldots, d$, where $g_{i}^{j}=1+g_{2 j-1}^{j-1}+$ $g_{2 j}^{j-1}+\cdots+g_{i-2}^{j-1}, h_{i}^{j}=h_{2 j-1}^{j-1}+h_{2 j}^{j-1}+\cdots+h_{i-2}^{j-1}, g_{i}^{1}=i-1, h_{i}^{1}=i-2$, and $g_{i}^{0}=h_{i}^{0}=1$.

Proof. (i) First, we take $b=0$, do induction on $j$ and show that $a_{2 j}^{j}=k$. For $j=0, a_{0}^{0}=k$, and for $j=1$, we have $a_{2}^{1}=a_{1}^{1}+a_{0}^{0}=k$, as $a_{1}^{1}=0$ from the hypothesis. We assume that the result is true up to $j-1$. Now $a_{2 j}^{j}=$ $a_{2 j-1}^{j}+a_{2 j-2}^{j-1}=0+k$, since $a_{2 j-1}^{j}=0$ from the hypothesis. Hence $a_{2 j}^{j}=k$, for every $j$. Next let $b>0$. For any fixed $j \geq 1$ we do induction on $b$. If $b=1$, $a_{2 j+1}^{j}=a_{2 j}^{j}+a_{2 j-1}^{j-1}=k+a_{2 j-1}^{j-1}$. We assume that the equation holds true up to $b-1$. Now $a_{2 j+b}^{j}=a_{2 j+b-1}^{j}+a_{2 j+b-2}^{j-1}=k+a_{2 j-1}^{j-1}+a_{2 j}^{j-1}+\cdots+a_{2 j+b-3}^{j-1}+a_{2 j+b-2}^{j-1}$. This proves the first part of the lemma.

For $(i i)$, we do induction on $j$ for any fixed $i \geq 2$. By $(i)$ of this Lemma we get, $a_{i}^{1}=k+a_{1}^{0}+a_{2}^{0}+\cdots+a_{i-2}^{0}=k+(i-2)(k-1)=(i-1) k-(i-2)=g_{i}^{1} k-h_{i}^{1}$. This proves the result for $j=1$. Let the equation be true upto $j-1$. Now $a_{i}^{j}=k+a_{2 j-1}^{j-1}+a_{2 j}^{j-1}+\cdots+a_{i-2}^{j-1}=k+\left(g_{2 j-1}^{j-1} k-h_{2 j-1}^{j-1}\right)+\left(g_{2 j}^{j-1} k-h_{2 j}^{j-1}\right)+\cdots+$ $\left(g_{i-2}^{j-1} k-h_{i-2}^{j-1}\right)=\left(1+g_{2 j-1}^{j-1}+g_{2 j}^{j-1}+\cdots+g_{i-2}^{j-1}\right) k-\left(h_{2 j-1}^{j-1}+h_{2 j}^{j-1}+\cdots+h_{i-2}^{j-1}\right)=$ $g_{i}^{j} k-h_{i}^{j}$. Hence the result. 
Theorem 2.2. Let $G$ be a minimal $(k, g)$-cage. The $i^{\text {th }}$ distance matrix $A_{i}$, $i=0,1,2, \ldots, d$, of $G$ can be expressed as:

$$
\begin{aligned}
A_{i}= & \frac{1}{c}\left[A^{i}-a_{i}^{1} A^{i-2}+(k-1) a_{i}^{2} A^{i-4}-(k-1)^{2} a_{i}^{3} A^{i-6}+\cdots+(-1)^{\left\lfloor\frac{i}{2}\right\rfloor}\right. \\
& \left.(k-1)^{\left\lfloor\frac{i}{2}\right\rfloor-1} a_{i}^{\left\lfloor\frac{i}{2}\right\rfloor} A^{i-2\left\lfloor\frac{i}{2}\right\rfloor}\right]
\end{aligned}
$$

where $a_{i}^{j}$ are as in Lemma 2.1, $c$ is $k$ for $i=d$ and $g$ even, and is 1 otherwise. Proof. We do induction on $i$. First, let $g$ be an odd integer. So intersection array of $G$ is $\{k, k-1, \ldots, k-1 ; 1,1, \ldots, 1\}$. From recurrence relation (2), we have $A A_{1}=c_{2} A_{2}+a_{1} A_{1}+b_{0} A_{0}, A_{0}=I$ and $A_{1}=A$. Since $a_{1}=0, c_{2}=1$, and $b_{0}=k$, we get $A^{2}=A_{2}+k I$. Then $A_{2}=A^{2}-k I=A^{2}-a_{2}^{1} I$. Thus equation (3) is true for $i=0,1,2$. Let us assume that it is true up to $d-1$. Then we consider $i=d$. Since $a_{d-1}=0, c_{d}=1$, and $b_{d-2}=k-1$, we have

$$
A A_{d-1}=c_{d} A_{d}+a_{d-1} A_{d-1}+b_{d-2} A_{d-2}=A_{d}+(k-1) A_{d-2},
$$

$$
\begin{aligned}
& A\left(A^{d-1}-a_{d-1}^{1} A^{d-3}+\cdots+(-1)^{\left\lfloor\frac{d-1}{2}\right\rfloor}(k-1)^{\left\lfloor\frac{d-1}{2}\right\rfloor-1} a_{d}^{\left\lfloor\frac{d-1}{2}\right\rfloor} A^{d-1-\left\lfloor\frac{d-1}{2}\right\rfloor}\right)=A_{d}+ \\
& (k-1)\left(A^{d-2}-a_{d-2}^{1} A^{d-4}+\cdots+(-1)^{\left\lfloor\frac{d-2}{2}\right\rfloor}(k-1)^{\left\lfloor\frac{d-2}{2}\right\rfloor-1} a_{d-2}^{\left\lfloor\frac{d-2}{2}\right\rfloor} A^{d-2-2\left\lfloor\frac{d-2}{2}\right\rfloor}\right) .
\end{aligned}
$$

Since for $d$ even, $\left\lfloor\frac{d-2}{2}\right\rfloor=\left\lfloor\frac{d-1}{2}\right\rfloor=\left\lfloor\frac{d}{2}\right\rfloor-1$, we get

$$
\begin{aligned}
A_{d}= & A^{d}-\left\{a_{d-1}^{1}+(k-1)\right\} A^{d-2}+(k-1)\left\{a_{d-1}^{2}+a_{d-2}^{1}\right\} A^{d-4}-\cdots+(-1)^{\left\lfloor\frac{d}{2}\right\rfloor} \\
& (k-1)^{\left\lfloor\frac{d}{2}\right\rfloor-1} a_{d-2}^{\left\lfloor\frac{d-2}{2}\right\rfloor} A^{d-2\left\lfloor\frac{d}{2}\right\rfloor} \\
= & A^{d}-a_{d}^{1} A^{d-2}+(k-1) a_{d}^{2} A^{d-4}-\cdots+(-1)^{\left\lfloor\frac{d}{2}\right\rfloor}(k-1)^{\left\lfloor\frac{d}{2}\right\rfloor-1} a_{d}^{\left\lfloor\frac{d}{2}\right\rfloor} A^{d-2\left\lfloor\frac{d}{2}\right\rfloor} .
\end{aligned}
$$

For $d$ odd, $\left\lfloor\frac{d-2}{2}\right\rfloor=\left\lfloor\frac{d}{2}\right\rfloor-1$ and $\left\lfloor\frac{d-1}{2}\right\rfloor=\left\lfloor\frac{d}{2}\right\rfloor$. So we get

$$
\begin{aligned}
A_{d}= & A^{d}-\left\{a_{d-1}^{1}+(k-1)\right\} A^{d-2}+(k-1)\left\{a_{d-1}^{2}+a_{d-2}^{1}\right\} A^{d-4}-\cdots+(-1)^{\left\lfloor\frac{d}{2}\right\rfloor} \\
& (k-1)^{\left\lfloor\frac{d}{2}\right\rfloor-1}\left\{a_{d-1}^{\left\lfloor\frac{d}{2}\right\rfloor}+a_{d-2}^{\left\lfloor\frac{d}{2}\right\rfloor-1}\right\} A^{d-2\left\lfloor\frac{d}{2}\right\rfloor} \\
= & A^{d}-a_{d}^{1} A^{d-2}+(k-1) a_{d}^{2} A^{d-4}-\cdots+(-1)^{\left\lfloor\frac{d}{2}\right\rfloor}(k-1)^{\left\lfloor\frac{d}{2}\right\rfloor-1} a_{d}^{\left\lfloor\frac{d}{2}\right\rfloor} A^{d-2\left\lfloor\frac{d}{2}\right\rfloor} .
\end{aligned}
$$

Hence the result holds true in this case.

Next, we consider that $g$ is even. The result holds true for $i=0,1,2, \ldots, d-$ 1 , because the intersection numbers agree with those in the case that $g$ is odd. Since $c_{d}=k$, by recurrence relation (2) we get

$$
\begin{aligned}
& A A_{d-1}=c_{d} A_{d}+a_{d-1} A_{d-1}+b_{d-2} A_{d-2}=k A_{d}+(k-1) A_{d-2}, \\
& A_{d}=\frac{1}{k}\left[A^{d}-a_{d}^{1} A^{d-2}+(k-1) a_{d}^{2} A^{d-4}-\cdots+(-1)^{\left\lfloor\frac{d}{2}\right\rfloor}(k-1)^{\left\lfloor\frac{d}{2}\right\rfloor-1} a_{d}^{\left\lfloor\frac{d}{2}\right\rfloor} A^{d-2\left\lfloor\frac{d}{2}\right\rfloor}\right] .
\end{aligned}
$$


In the theorem below we find polynomials of degree $\left\lfloor\frac{g}{2}\right\rfloor$ which give all distance eigenvalues of minimal $(k, g)$-cages when the variable is substituted by adjacency eigenvalues.

Theorem 2.3. If $\lambda$ is an eigenvalue of a minimal $(k, g)$-cage $G$ then $p(\lambda)$ is a distance eigenvalue of $G$ with the same multiplicity as that of $\lambda$, where $p(x)$ is given below.

$$
p(x)= \begin{cases}\sum_{i=0}^{d-1}\left[i+\sum_{j=1}^{\left\lfloor\frac{d-i-1}{2}\right\rfloor}(-1)^{j}(i+2 j)(k-1)^{j-1} a_{i+2 j}^{j}\right] x^{i}+\frac{d}{k}\left[A^{d}+\right. & \\ \left\lfloor\frac{d}{2}\right\rfloor & \\ \left.\sum_{i=1}^{d-i \geq 3}(-1)^{i}(k-1)^{i-1} a_{d}^{i} x^{d-2 i}\right], & \text { even } \\ x, & \text { for } g=3 \\ 2 x^{2}+x-2 k, & \text { for } g=5\end{cases}
$$

where $a_{i}^{j}$ are as in Lemma 2.1.

Proof. Here we represent $D(G)$ as a polynomial, $p(A)$, of the adjacency matrix $A$ of $G$ and then the theorem follows from a basic result that "If $\lambda$ is an eigenvalue of $A$ then $p(\lambda)$ is an eigenvalue of $p(A)$ with the same multiplicity as that of $\lambda . "$ First, we consider that $g$ is an even integer. From Theorem 2.2 and equation (11), the distance matrix $D$ of a minimal $(k, g)$-cage $G$ can be written as below:

$$
\begin{aligned}
D & =A_{1}+2 A_{2}+3 A_{3} \cdots+d A_{d} \\
& =A+2\left(A^{2}-a_{2}^{1} I\right)+3\left(A^{3}-a_{3}^{1} A\right)+\cdots+(d-1)\left[A^{d-1}-a_{d-1}^{1} A^{d-3}+\cdots+\right. \\
& \left.(-1)^{\left\lfloor\frac{d-1}{2}\right\rfloor}(k-1)^{\left\lfloor\frac{d-1}{2}\right\rfloor-1} a_{d-1}^{\left\lfloor\frac{d-1}{2}\right\rfloor} A^{d-1-2\left(\left\lfloor\frac{d-1}{2}\right\rfloor\right)}\right]+\frac{d}{k}\left[A^{d}-a_{d}^{1} A^{d-2}+\cdots+(-1)^{\left\lfloor\frac{d}{2}\right\rfloor}\right. \\
& \left.(k-1)^{\left\lfloor\frac{d}{2}\right\rfloor-1} a_{d}^{\left\lfloor\frac{d}{2}\right\rfloor} A^{d-2\left\lfloor\frac{d}{2}\right\rfloor}\right]
\end{aligned}
$$

If $d$ is even then $\left\lfloor\frac{d-i-2}{2}\right\rfloor=\left\lfloor\frac{d-i-1}{2}\right\rfloor$ for even $i$ and $\left\lfloor\frac{d-i}{2}\right\rfloor=\left\lfloor\frac{d-i-1}{2}\right\rfloor$ for odd $i$, $i=1, \ldots, d-1$. So we get,

$$
\begin{aligned}
D & =\left[-2 a_{2}^{1}+4(k-1) a_{4}^{2}-\cdots+(-1)^{\left\lfloor\frac{d-2}{2}\right\rfloor}(d-2)(k-1)^{\left\lfloor\frac{d-2}{2}\right\rfloor-1} a_{d-2}^{\left\lfloor\frac{d-2}{2}\right\rfloor}\right]+\left[1-3 a_{3}^{1}\right. \\
& \left.+\cdots+(-1)^{\left\lfloor\frac{d-1}{2}\right\rfloor}(d-1)(k-1)^{\left\lfloor\frac{d-1}{2}\right\rfloor-1} a_{d-1}^{\left\lfloor\frac{d-1}{2}\right\rfloor}\right] A+\cdots+\left[(d-3)-(d-1) a_{d-1}^{1}\right] \\
& A^{d-3}+(d-2) A^{d-2}+(d-1) A^{d-1}+\frac{d}{k}\left[A^{d}-a_{d}^{1} A^{d-2}+(k-1) a_{d}^{2} A^{d-4}-\cdots+\right. \\
& \left.(-1)^{\left\lfloor\frac{d}{2}\right\rfloor}(k-1)^{\left\lfloor\frac{d}{2}\right\rfloor-1} a_{d}^{\left\lfloor\frac{d}{2}\right\rfloor} A^{d-2\left\lfloor\frac{d}{2}\right\rfloor}\right]
\end{aligned}
$$




$$
\begin{aligned}
= & \sum_{i=0}^{d-1}\left[i+\sum_{\substack{j=1 \\
d-i \geq 3}}^{\left\lceil\frac{d-i-1}{2}\right\rceil}(-1)^{j}(i+2 j)(k-1)^{j-1} a_{i+2 j}^{j}\right] A^{i}+\frac{d}{k}\left[A^{d}+\sum_{i=1}^{\left\lfloor\frac{d}{2}\right\rfloor}(-1)^{i}(k-1)^{i-1}\right. \\
& \left.a_{d}^{i} A^{d-2 i}\right]
\end{aligned}
$$

For $d$ odd, we get

$$
\begin{aligned}
D= & {\left[-2 a_{2}^{1}+4(k-1) a_{4}^{2}-\cdots+(-1)^{\left\lfloor\frac{d-1}{2}\right\rfloor}(d-1)(k-1)^{\left\lfloor\frac{d-1}{2}\right\rfloor-1} a_{d-1}^{\left\lfloor\frac{d-1}{2}\right\rfloor}\right]+[1-3} \\
& \left.a_{3}^{1}+\cdots+(-1)^{\left\lfloor\frac{\lfloor-2}{2}\right\rfloor}(d-2)(k-1)^{\left\lfloor\frac{d-2}{2}\right\rfloor-1} a_{d-2}^{\left\lfloor\frac{d-2}{2}\right\rfloor}\right] A+\cdots+[(d-3)-(d-1) \\
& \left.a_{d-1}^{1}\right] A^{d-3}+(d-2) A^{d-2}+(d-1) A^{d-1}+\frac{d}{k}\left[A^{d}-a_{d}^{1} A^{d-2}+\cdots+(-1)^{\left\lfloor\frac{d}{2}\right\rfloor}\right. \\
& \left.(k-1)^{\left\lfloor\frac{d}{2}\right\rfloor-1} a_{d}^{\left\lfloor\frac{d}{2}\right\rfloor} A^{d-2\left\lfloor\frac{d}{2}\right\rfloor}\right] \\
= & \sum_{i=0}^{d-1}\left[i+\sum_{j=1}^{\left\lfloor\frac{d-i-1}{2}\right\rfloor}(-1)^{j}(i+2 j)(k-1)^{j-1} a_{i+2 j}^{j}\right] A^{i}+\frac{d}{k}\left[A^{d}+\sum_{i=1}^{\left\lfloor\frac{d}{2}\right\rfloor}(-1)^{i}\right. \\
& \left.(k-1)^{i-1} a_{d}^{i} A^{d-2 i}\right]
\end{aligned}
$$

In the above, for both $d$ even and odd, $D$ is expressed as a polynomial of the adjacency matrix $A$ of $G$. We take this polynomial as $p(A)$ and obtain the result.

If $g$ is an odd integer then by Lemma 1.4, $g=3$ or 5 . For $g=3$ the value of $d$ is 1 . So, by equation (11) we have $D=p(A)=A$, and so is the result. Now for $g=5$, the value of $d$ is 2. Applying equation (11) and Theorem 2.2 we have, $D=A_{1}+2 A_{2}=A+2\left(A^{2}-a_{2}^{1} I\right)=A+2\left(A^{2}-k I\right)=2 A^{2}+A-2 k I$, and hence the result.

Theorem 2.4. Every minimal $(k, g)$-cage, $k \geq 2$, with diameter $d$ has $d+1$ distinct distance eigenvalues.

Proof. For $k=2$ the minimal $(k, g)$-cages are cycles, and the result follows by [14].

Case 1. In this case we consider that $g$ is an even integer. For $d$ even, applying Lemma 2.1 and Theorem 2.3 the distance matrix of the minimal 
$(k, g)$-cage can be written as,

$$
\begin{aligned}
& D=p(A)=A+2\left(A^{2}-a_{2}^{1} I\right)+\cdots+(d-2)\left[A^{d-2}-a_{d-2}^{1} A^{d-4}+\cdots+(-1)^{\left\lfloor\frac{d-2}{2}\right\rfloor-1}\right. \\
& (k-1)^{\left\lfloor\frac{d-2}{2}\right\rfloor-2} a_{d-2}^{\left\lfloor\frac{d-2}{2}\right\rfloor-1} A^{d-2-2\left(\left\lfloor\frac{d-2}{2}\right\rfloor-1\right)}+(-1)^{\left\lfloor\frac{d-2}{2}\right\rfloor}(k-1)^{\left\lfloor\frac{d-2}{2}\right\rfloor-1} a_{d-2}^{\left\lfloor\frac{d-2}{2}\right\rfloor} \\
& \left.A^{d-2-2\left(\left\lfloor\frac{d-2}{2}\right\rfloor\right)}\right]+(d-1)\left[A^{d-1}-a_{d-1}^{1} A^{d-3}+\cdots+(-1)^{\left\lfloor\frac{d-1}{2}\right\rfloor-1}(k-1)^{\left\lfloor\frac{d-1}{2}\right\rfloor-2}\right. \\
& \left.a_{d-1}^{\left\lfloor\frac{d-1}{2}\right\rfloor-1} A^{d-1-2\left(\left\lfloor\frac{d-1}{2}\right\rfloor-1\right)}+(-1)^{\left\lfloor\frac{d-1}{2}\right\rfloor}(k-1)^{\left\lfloor\frac{d-1}{2}\right\rfloor-1} a_{d-1}^{\left\lfloor\frac{d-1}{2}\right\rfloor} A^{d-1-2\left(\left\lfloor\frac{d-1}{2}\right\rfloor\right)}\right]+ \\
& \frac{d}{k}\left[A^{d}-a_{d}^{1} A^{d-2}+\cdots+(-1)^{\left\lfloor\frac{d}{2}\right\rfloor-1}(k-1)^{\left\lfloor\frac{d}{2}\right\rfloor-2} a_{d}^{\left\lfloor\frac{d}{2}\right\rfloor-1} A^{d-2\left(\left\lfloor\frac{d}{2}\right\rfloor-1\right)}+(-1)^{\left\lfloor\frac{d}{2}\right\rfloor}\right. \\
& \left.(k-1)^{\left\lfloor\frac{d}{2}\right\rfloor-1} a_{d}^{\left\lfloor\frac{d}{2}\right\rfloor} A^{d-2\left\lfloor\frac{d}{2}\right\rfloor}\right] \\
& =A+2\left(A^{2}-k I\right)+\cdots+(d-2)\left[A^{d-2}-\left(g_{d-2}^{1} k-h_{d-2}^{1}\right) A^{d-4}+\cdots+(-1)^{\left\lfloor\frac{d-2}{2}\right\rfloor}\right. \\
& \left.k \sum_{i=0}^{\left\lfloor\frac{d-2}{2}\right\rfloor-1}(-1)^{i}\left(\begin{array}{c}
\left\lfloor\frac{d-2}{2}\right\rfloor-1 \\
i
\end{array}\right) k^{\left\lfloor\frac{d-2}{2}\right\rfloor-1-i}\right]+(d-1)\left[A^{d-1}-\left(g_{d-1}^{1} k-h_{d-1}^{1}\right) A^{d-3}\right. \\
& \left.+\cdots+(-1)^{\left\lfloor\frac{d-1}{2}\right\rfloor} \sum_{i=0}^{\left\lfloor\frac{d-1}{2}\right\rfloor-1}(-1)^{i}\left(\begin{array}{c}
\left\lfloor\frac{d-1}{2}\right\rfloor-1 \\
i
\end{array}\right) k^{\left\lfloor\frac{d-1}{2}\right\rfloor-1-i}\left(g_{d-1}^{\left\lfloor\frac{d-1}{2}\right\rfloor} k-h_{d-1}^{\left\lfloor\frac{d-1}{2}\right\rfloor}\right) A\right]+ \\
& \frac{d}{k}\left[A^{d}-\left(g_{d}^{1} k-h_{d}^{1}\right) A^{d-2}+\cdots+(-1)^{\left\lfloor\frac{d}{2}\right\rfloor} k \sum_{i=0}^{\left\lfloor\frac{d}{2}\right\rfloor-1}(-1)^{i}\left(\begin{array}{c}
\left\lfloor\frac{d}{2}\right\rfloor-1 \\
i
\end{array}\right) k^{\left\lfloor\frac{d}{2}\right\rfloor-1-i}\right] \\
& =\frac{1}{k}\left[d\left\{A^{d}+h_{d}^{1} A^{d-2}+\cdots+h_{d}^{\left\lfloor\frac{d}{2}\right\rfloor-1} A^{2}\right\}+\left\{A+2 A^{2}+3\left(A^{3}+h_{3}^{1} A\right)+\cdots+(d\right.\right. \\
& -1)\left(A^{d-1}+h_{d-1}^{1} A^{d-3}+\cdots+h_{d-1}^{\left\lfloor\frac{d-1}{2}\right\rfloor} A\right)-d\left(g_{d}^{1} A^{d-2}+\left(g_{d}^{2}+h_{d}^{2}\right) A^{d-4}+\left(g_{d}^{3}\right.\right. \\
& \left.\left.\left.+2 h_{d}^{3}\right) A^{d-6}+\cdots+\left(g_{d}^{\left\lfloor\frac{d}{2}\right\rfloor-1}+\left(\left\lfloor\frac{d}{2}\right\rfloor-1\right) h_{d}^{\left\lfloor\frac{d}{2}\right\rfloor-1}\right) A^{2}+1\right)\right\} k+\cdots+(-1)^{\left\lfloor\frac{d}{2}\right\rfloor-1} \\
& \left.\left\{(d-2)+g_{d-1}^{\left\lfloor\frac{d-1}{2}\right\rfloor}(d-1) A-d\right\} k^{\left\lfloor\frac{d}{2}\right\rfloor}\right],
\end{aligned}
$$

since for $d$ even $\left\lfloor\frac{d}{2}\right\rfloor-1=\left\lfloor\frac{d-1}{2}\right\rfloor=\left\lfloor\frac{d-2}{2}\right\rfloor$. If possible let there be two distinct eigenvalues $\lambda_{i} \neq \lambda_{j}$ of the minimal $(k, g)$-cage such that $p\left(\lambda_{i}\right)=p\left(\lambda_{j}\right)$. This implies $k p\left(\lambda_{i}\right)=k p\left(\lambda_{j}\right)$ (since $k \neq 0$ ). Now equating the coefficients of $k^{\left\lfloor\frac{d}{2}\right\rfloor}$, we get $(d-2)+(d-1) g_{d-1}^{\left\lfloor\frac{d-1}{2}\right\rfloor} \lambda_{i}-d=(d-2)+(d-1) g_{d-1}^{\left\lfloor\frac{d-1}{2}\right\rfloor} \lambda_{j}-d$, which gives $\lambda_{i}=\lambda_{j}$, a contradiction. This proves that a minimal $(k, g)$-cage has $d+1$ distinct distance eigenvalues for $d$ and $g$ both even.

If $d$ is odd and $g$ is even then by Lemma 1.4 we get that $g$ is equal to 6. By Lemma [1.5] all distinct eigenvalues of the minimal $(k, 6)$-cage are $\pm k, \pm \sqrt{k-1}$. Then the distance matrix of the minimal $(k, 6)$-cage can be written as $D=p(A)=\frac{3}{k} A^{3}+2 A^{2}+\frac{3-5 k}{k} A-2 k I$. If possible let there exist two distinct eigenvalues $\lambda_{i} \neq \lambda_{j}$ of minimal $(k, 6)$-cage such 
that $p\left(\lambda_{i}\right)=p\left(\lambda_{j}\right)$. Then $k p\left(\lambda_{i}\right)=k p\left(\lambda_{j}\right)$ (since $k \neq 0$ ). This implies $-2 k^{2}+\left(2 \lambda_{i}^{2}-5 \lambda_{i}\right) k+3\left(\lambda_{i}^{3}+\lambda_{i}\right)=-2 k^{2}+\left(2 \lambda_{j}^{2}-5 \lambda_{j}\right) k+3\left(\lambda_{j}^{3}+\lambda_{j}\right)$. Equating the coefficients of $k$ we get $2\left(\lambda_{i}^{2}-\lambda_{j}^{2}\right)-5\left(\lambda_{i}-\lambda_{j}\right)=0$, and then $\lambda_{i}+\lambda_{j}=\frac{5}{2}$. Since $k$ is the largest adjacency eigenvalue, $p(k)$ is the largest distance eigenvalue [1]. Thus both $\lambda_{i}$ and $\lambda_{j}$ are different from $k$. Then $\lambda_{i}, \lambda_{j} \in\{-k, \pm \sqrt{k-1}\}$. Now $\lambda_{i}+\lambda_{j}=-k \pm \sqrt{k-1}=\frac{5}{2}$, that is $4 k^{2}+16 k+29=0$. But this equation does not give any integer solution and since $k$ is an integer, this leads to a contradiction. Hence a minimal $(k, 6)$-cage has 4 distinct distance eigenvalues.

Case 2. In this case we consider that $g$ is an odd integer. A minimal $(k, 3)$-cage is a complete graph $K_{n}$ and its distinct distance eigenvalues are $n-1,-1$. Now for $g=5$, all distinct eigenvalues of the minimal $(k, 5)$-cage are $k, \frac{-1 \pm \sqrt{4 k-3}}{2}$. Then from Theorem 2.3 the distance matrix of this graph can be written as $D=p(A)=2 A^{2}+A-2 k I$. If possible let there exist two distinct eigenvalues $\lambda_{i} \neq \lambda_{j}$ of minimal $(k, 5)$-cage such that $p\left(\lambda_{i}\right)=p\left(\lambda_{j}\right)$. This implies $2\left(\lambda_{i}^{2}-\lambda_{j}^{2}\right)+\left(\lambda_{i}-\lambda_{j}\right)=0$, and then $\lambda_{i}+\lambda_{j}=-\frac{1}{2}$. Since $k$ is the largest adjacency eigenvalue, $p(k)$ is the largest distance eigenvalue [1]. Thus both $\lambda_{i}$ and $\lambda_{j}$ are different from $k$. Then $\lambda_{i}, \lambda_{j} \in\left\{\frac{-1 \pm \sqrt{4 k-3}}{2}\right\}$ and $\lambda_{i}+\lambda_{j}=-1$, a contradiction. Thus a minimal $(k, 5)$-cage has 3 distinct distance eigenvalues. This proves the theorem.

Remark 2.1. Theorem 2.4 supplies a class of graphs to the answer of the problem "Characterize distance regular graphs with diameter $d$ and having exactly $d+1$ distinct $D$-eigenvalues", asked by Atik and Panigrahi [5].

A minimal $(k, 3)$-cage is a complete graph and its distance spectrum is mentioned in Theorem 2.4. Minimal $(k, 4)$-cages and $(2, g)$-cages are complete bipartite graphs and cycles respectively, and their distance spectrum can be found in [18] and [14]. So in the theorem below we present the distance spectrum of minimal $(k, g)$-cages, $k \geq 3$ and $g \geq 5$, by applying Lemma 1.5 and Theorems 2.2 and 2.3 .

Theorem 2.5. 1. The distance matrix of a minimal $(k, 5)$-cage is $D=$ $-2 k I+A+2 A^{2}$.

(a) The distance matrix of the minimal $(3,5)$-cage (Petersen graph) is $D=-6 I+A+2 A^{2}$, and its distance spectrum is $\left\{15,-3^{(5)}, 0^{(4)}\right\}$.

(b) The distance matrix of the minimal $(7,5)$-cage (Hoffman-Singleton graph) is $D=-14 I+A+2 A^{2}$, and its distance spectrum is $\left\{91,-4^{(28)}, 1^{(21)}\right\}$.

(c) The distance matrix of the minimal $(57,5)$-cage (if exists) is $D=$ $-114 I+A+2 A^{2}$, and its distance spectrum is $\left\{6441,-9^{(1729)}, 6^{(1520)}\right\}$. 
2. The distance matrix of a minimal $(k, 6)$-cage is $D=\frac{3}{k} A^{3}+2 A^{2}-$ $\frac{5 k-3}{k} A-2 k I$, and its distance spectrum is $\left\{5 k^{2}-7 k+3,-k^{2}+3 k-\right.$ $\left.3,(-2(1+\sqrt{k-1}))^{\left(m_{\sqrt{k-1}}\right)},(-2(1-\sqrt{k-1}))^{\left(m_{-\sqrt{k-1}}\right)}\right\}$, where $m_{ \pm \sqrt{k-1}}=$ $\frac{n k(k-1)}{2\left(k^{2}-k+1\right)}$.

(Heawood graph is a minimal $(3,6)$-cage and its distance matrix is $D=$ $A^{3}+2 A^{2}-4 A-6 I$, and its distance spectrum is $\left\{27,-3,(-2(1+\sqrt{2}))^{(6)}\right.$, $\left.\left.(-2(1-\sqrt{2}))^{(6)}\right\}\right)$.

3. The distance matrix of a minimal $(k, 8)$-cage is $D=\frac{4}{k} A^{4}+3 A^{3}+$ $\frac{8-10 k}{k} A^{2}-(6 k-4) A+(2 k-4) I$, and its distance spectrum is $\left\{7 k^{3}-16 k^{2}+\right.$ $14 k-4, k^{3}-4 k^{2}+6 k-4,(2 k-4)^{\left(m_{0}\right)},(-2(k+\sqrt{2(k-1)}))^{(m \sqrt{2(k-1)})},(-2(k-$ $\left.\sqrt{2(k-1)}))^{\left(m_{-\sqrt{2(k-1)}}\right)}\right\}$, where $m_{0}=\frac{n(k-1)}{2 k}, m_{ \pm \sqrt{2(k-1)}}=\frac{n k(k-1)}{4\left(k^{2}-2 k+2\right)}$.

( Levi graph is a minimal $(3,8)$-cage and its distance matrix is $D=$ $\frac{4}{3} A^{4}+3 A^{3}-\frac{22}{3} A^{2}-14 A+2 I$, and its distance spectrum is $\left\{83,5,2^{(10)}\right.$, $\left.\left.-10^{(9)},-2^{(9)}\right\}\right)$.

4. The distance matrix of a minimal $(k, 12)$-cage is $D=\frac{6}{k} A^{6}+5 A^{5}+$ $\frac{24-26 k}{k} A^{4}-(20 k-18) A^{3}+\frac{24 k^{2}-44 k+18}{k} A^{2}+\left(15 k^{2}-26 k+9\right) A-\left(2 k^{2}-\right.$ $6 k+6) I$, and its distance spectrum is $\left\{11 k^{5}-46 k^{4}+81 k^{3}-72 k^{2}+\right.$ $33 k-6, k^{5}-6 k^{4}+15 k^{3}-20 k^{2}+15 k-6,\left(-2 k^{2}+6 k-6\right)^{\left(m_{0}\right)},(2(k-$ $2)(k+\sqrt{k-1}))^{\left(m_{\sqrt{k-1}}\right)},(2(k-2)(k-\sqrt{k-1}))^{\left(m_{-\sqrt{k-1}}\right)},(-2 k(k+$ $\left.\sqrt{3(k-1)}))^{\left(m_{\sqrt{3(k-1)}}\right.},(-2 k(k-\sqrt{3(k-1)}))^{\left(m_{-\sqrt{3(k-1)}}\right)}\right\}$, where $m_{0}=$ $\frac{n(k-1)}{3 k}, m_{ \pm \sqrt{k-1}}=\frac{n k(k-1)}{4\left(k^{2}-k+1\right)}$, and $m_{ \pm \sqrt{3(k-1)}}=\frac{n k(k-1)}{12\left(k^{2}-3 k+3\right)}$.

\section{Distance spectrum of some distance bireg- ular graphs}

In the next theorem we show that every DBR graph is a 2-partitioned transmission regular graph.

Theorem 3.1. All distance biregular (DBR) graphs are 2-partitioned transmission regular graphs.

Proof. Let $G$ be a DBR graph with partite sets $V_{1}$ and $V_{2}$. Each vertex $u$ in $V_{1}$ has $l_{i}$, a constant, number of vertices at distance $i$. From $u$, even distance vertices are situated in $V_{1}$ and odd distance vertices are in $V_{2}$. Thus the number of vertices of even and odd distances is constant from each vertex $u \in V_{1}$. So we get, $q_{11}=\sum_{v \in V_{1}} d(u, v)=\sum_{i=0}^{\left\lfloor\frac{d_{1}}{2}\right\rfloor} 2 i l_{2 i}$ and $q_{12}=\sum_{v \in V_{2}} d(u, v)=$ 
$\sum_{i=0}^{\left\lfloor\frac{d_{1}}{2}\right\rfloor}(2 i+1) l_{2 i+1}$ are constants, where $d_{1}=\max \left\{d(x, y): x \in V_{1}, y \in V(G)\right\}$. Similarly the sum of distances from each $w \in V_{2}, q_{21}=\sum_{v \in V_{1}} d(w, v)=\sum_{i=0}^{\left\lfloor\frac{d_{2}}{2}\right\rfloor} 2 i l_{2 i}^{\prime}$ and $q_{22}=\sum_{v \in V_{2}} d(w, v)=\sum_{i=0}^{\left\lfloor\frac{d_{2}}{2}\right\rfloor}(2 i+1) l_{2 i+1}^{\prime}$ are constants, where $l_{i}^{\prime}$ is the number of vertices at distance $i$ from $w$ and $d_{2}=\max \left\{d(x, y): x \in V_{2}, y \in V(G)\right\}$. Hence the result.

The larger root of the quotient matrix $Q=\left(\begin{array}{ll}q_{11} & q_{12} \\ q_{21} & q_{22}\end{array}\right)$ is the distance spectral radius of any DBR graphs by Lemma 1.3. The subdivision graph $S(G)$ of a minimal $(k, g)$-cage $G$ is a DBR graph [16]. So applying Theorem 3.1 we determine distance spectral radius of these graphs. Unless otherwise stated, in the remaining of the paper $\left(V_{1}, V_{2}\right)$ is taken as the vertex partition of subdivision of a minimal $(k, g)$-cage $G$, where $V_{1}=V(G)$ and $V_{2}$ is the set of all new vertices inserted on edges of $G$.

Theorem 3.2. Let $g$ be an even integer and $G$ be a minimal $(k, g)$-cage with diameter $d$. The distance spectral radius of the subdivision graph $S(G)$ is $(3 k-2) S_{1}^{\prime}+d k(k-1)^{d-1}+$ $\sqrt{(k-2)^{2} S_{1}^{\prime 2}+2 k S_{2}^{\prime 2}+2 d(k-2)^{2}(k-1)^{d-1} S_{1}^{\prime}+d^{2}(k-2)^{2}(k-1)^{2 d-2}}$, where $S_{1}^{\prime}=\frac{1}{(2-k)^{2}}\left[(k d-2 d-k+1)(k-1)^{d-1}+1\right]$ and $S_{2}^{\prime}=\frac{1}{(2-k)^{2}}[(2 k d-4 d-k)(k-$ $\left.1)^{d}+k\right]$.

Proof. We know that [16] the intersection array of any vertex $u \in V_{1}$ is $\{k, 1, k-1,1, \ldots, k-1,1 ; 1,1,1,1, \ldots, 1, k\}$ and the intersection array of any vertex $v \in V_{2}$ is $\{2, k-1,1, k-1, \cdots, 1, k-1 ; 1,1,1,1, \cdots, 1,2\}$. Since $S(G)$ is obtained by inserting a new vertex in every edge and diameter of $G$ is $d=\left\lfloor\frac{g}{2}\right\rfloor$, applying Theorem 3.1 we have $l_{0}=1, l_{2 i}=l_{2 i-1}=k(k-1)^{i-1}$, $i=1,2, \ldots, d-1, l_{2 d-1}=k(k-1)^{d-1}, l_{2 d}=(k-1)^{d-1}, l_{1}^{\prime}=2, l_{2 i}^{\prime}=l_{2 i+1}^{\prime}=$ $2(k-1)^{i}, i=1,2, \ldots, d-1$, and $l_{2 d}^{\prime}=(k-1)^{d}$. Thus

$$
\begin{aligned}
q_{11} & =\sum_{i=0}^{d} 2 i l_{2 i}=0+2 k+4 k(k-1)+\cdots+(2 d-2) k(k-1)^{d-2}+2 d(k-1)^{d-1} \\
& =2 k\left[1+2(k-1)+3(k-1)^{2}+\cdots+(d-1)(k-1)^{d-2}\right]+2 d(k-1)^{d-1} \\
& =2 k S_{1}^{\prime}+2 d(k-1)^{d-1}
\end{aligned}
$$


where $S_{1}^{\prime}=1+2(k-1)+3(k-1)^{2}+\cdots+(d-1)(k-1)^{d-2}=\frac{1}{(2-k)^{2}}[(k d-$ $\left.2 d-k+1)(k-1)^{d-1}+1\right]$.

$$
\begin{aligned}
q_{12} & =\sum_{i=0}^{d-1}(2 i+1) l_{2 i+1}=k+3 k(k-1)+5 k(k-1)^{2}+\cdots+(2 d-1) k(k-1)^{d-1} \\
& =k\left[1+3(k-1)+5(k-1)^{2}+\cdots+(2 d-1)(k-1)^{d-1}\right]=k S_{2}^{\prime},
\end{aligned}
$$

where $S_{2}^{\prime}=1+3(k-1)+5(k-1)^{2}+\cdots+(2 d-1)(k-1)^{d-1}=\frac{1}{(2-k)^{2}}[(2 k d-$ $\left.4 d-k)(k-1)^{d}+k\right]$.

$$
\begin{aligned}
q_{21}= & \sum_{i=0}^{d-1}(2 i+1) l_{2 i+1}^{\prime}=2+3 \times 2(k-1)+5 \times 2(k-1)^{2}+\cdots+(2 d-1) \times 2 \\
& (k-1)^{d-1}=2 S_{2}^{\prime} . \\
q_{22}= & \sum_{i=0}^{d} 2 i l_{2 i}^{\prime}=2 \times 2(k-1)+4 \times 2(k-1)^{2}+6 \times 2(k-1)^{3}+\cdots+(2 d-2) \\
& \times 2(k-1)^{d-1}+2 d \times(k-1)^{d}=4(k-1) S_{1}^{\prime}+2 d(k-1)^{d} .
\end{aligned}
$$

Thus $Q=\left(\begin{array}{ll}q_{11} & q_{12} \\ q_{21} & q_{22}\end{array}\right)$ is a quotient matrix of the distance matrix of $S(G)$ when $g$ is even. The characteristic polynomial of $Q$ is $x^{2}-\left\{2(3 k-2) S_{1}^{\prime}+\right.$ $\left.2 d k(k-1)^{d-1}\right\} x+8 k(k-1) S_{1}^{\prime 2}-2 k S_{2}^{\prime 2}+4 d(k+2)(k-1)^{d} S_{1}^{\prime}+4 d^{2}(k-1)^{2 d-1}=0$, and its larger root $(3 k-2) S_{1}^{\prime}+d k(k-1)^{d-1}+$ $\sqrt{(k-2)^{2} S_{1}^{\prime 2}+2 k S_{2}^{\prime 2}+2 d(k-2)^{2}(k-1)^{d-1} S_{1}^{\prime}+d^{2}(k-2)^{2}(k-1)^{2 d-2}}$, where $S_{1}^{\prime}=\frac{1}{(2-k)^{2}}\left[(k d-2 d-k+1)(k-1)^{d-1}+1\right]$ and $S_{2}^{\prime}=\frac{1}{(2-k)^{2}}[(2 k d-4 d-k)(k-$ $\left.1)^{d}+k\right]$ is the distance spectral radius of $S(G)$ by Lemma 1.3 .

By Lemma 1.4, if $g$ is an odd integer then minimal $(k, g)$-cages exist only for $g=3$ and 5 . So in the next theorem we determine distance spectral radius of subdivision of minimal $(k, g)$-cages for these two cases only.

Theorem 3.3. Let $g$ be an odd integer and $G$ be a minimal $(k, g)$-cage. The distance spectral radius of the subdivision graph $S(G)$ is,

$\lambda_{1}(S(G))= \begin{cases}\frac{1}{2}\left[2 k^{2}+\sqrt{2 k(2 k+1)\left(k^{2}+1\right)}\right], & \text { if } g=3 \\ \frac{1}{2}\left[\left(3 k^{3}+k-2\right)+\sqrt{9 k^{6}+2 k^{5}+14 k^{4}-40 k^{3}+41 k^{2}-18 k+4}\right], & \text { if } g=5\end{cases}$

Proof. First, let $g$ be equal to 3 . We know that [16] intersection array of any vertex in $V_{1}$ is $\{k, 1, k-1 ; 1,1,2\}$ and the intersection array of any vertex in $V_{2}$ is $\{2, k-1,1, k-2 ; 1,1,2,2\}$. By Lemma 1.1 we have, $l_{0}=1$, $l_{1}=l_{2}=k, l_{3}=\frac{1}{2} k(k-1), l_{0}^{\prime}=1, l_{1}^{\prime}=2, l_{2}^{\prime}=2(k-1), l_{3}^{\prime}=(k-1)$, and 
$l_{4}^{\prime}=\frac{1}{2}(k-1)(k-2)$. Thus $q_{11}=2 k, q_{12}=k+\frac{3}{2} k(k-1)=\frac{1}{2} k(3 k-1)$, $q_{21}=2+3(k-1)=3 k-1, q_{22}=2 \times 2(k-1)+4 \times \frac{1}{2}(k-1)(k-2)=2 k(k-1)$. So $Q=\left(\begin{array}{ll}q_{11} & q_{12} \\ q_{21} & q_{22}\end{array}\right)$ is a quotient matrix of the distance matrix of $S(G)$. The characteristic polynomial of $Q$ is $x^{2}-2 k^{2} x-\frac{1}{2} k(k+1)^{2}=0$, and its larger root $\frac{1}{2}\left[2 k^{2}+\sqrt{2 k(2 k+1)\left(k^{2}+1\right)}\right]$ is the distance spectral radius of $S(G)$ by Lemma 1.3.

Next we take $g=5$. Intersection array of any vertex in $V_{1}$ is $\{k, 1, k-$ $1,1, k-1 ; 1,1,1,1,2\}$ and the intersection array of any vertex in $V_{2}$ is $\{2, k-$ $1,1, k-1,1, k-2 ; 1,1,1,1,2,2\}$. By Lemma 1.1 we have, $l_{0}=1, l_{1}=l_{2}=k$, $l_{3}=l_{4}=k(k-1), l_{5}=\frac{1}{2} k(k-1)^{2}, l_{0}^{\prime}=1, l_{1}^{\prime}=2, l_{2}^{\prime}=l_{3}^{\prime}=2(k-1)$, $l_{4}^{\prime}=2(k-1)^{2}, l_{5}^{\prime}=(k-1)^{2}$, and $l_{6}^{\prime}=\frac{1}{2}(k-1)^{2}(k-2)$. Thus $q_{11}=$ $2 k+4 k(k-1)=2 k(2 k-1), q_{12}=k+3 k(k-1)+\frac{5}{2} k(k-1)^{2}=\frac{1}{2} k\left(5 k^{2}-4 k+1\right)$, $q_{21}=2+3 \times 2(k-1)+5 \times(k-1)^{2}=\left(5 k^{2}-4 k+1\right), q_{22}=2 \times 2(k-1)+$ $4 \times 2(k-1)^{2}+6 \times \frac{1}{2}(k-1)^{2}(k-2)=(k-1)\left(3 k^{2}-k+2\right)$.

So $Q=\left(\begin{array}{ll}q_{11} & q_{12} \\ q_{21} & q_{22}\end{array}\right)$ is a quotient matrix of the distance matrix of $S(G)$. The characteristic polynomial of $Q$ is $x^{2}-\left(3 k^{3}+k-2\right) x-\frac{1}{2} k\left(k^{4}+4 k^{3}-14 k^{2}+\right.$ $20 k-7)=0$, and its larger root $\frac{1}{2}\left[\left(3 k^{3}+k-2\right)+\sqrt{9 k^{6}+2 k^{5}+14 k^{4}-40 k^{3}+41 k^{2}-18 k+4}\right]$ is the distance spectral radius of $S(G)$ by Lemma 1.3 .

Example 3.1. We know that the Heawood graph is the minimal $(3,6)$ cage. By Theorem 3.2, $Q=\left(\begin{array}{ll}54 & 81 \\ 54 & 88\end{array}\right)$ is a quotient matrix of the distance matrix of subdivision of Heawood graph, and its characteristic polynomial is $x^{2}-142 x+378$. So the distance spectral radius of subdivision of Heawood graph is $71+\sqrt{4663}$. We also compute the distance characteristic polynomial of subdivision of Heawood graph, which is $\left(x^{2}-142 x+378\right)(x+2)^{8}(x+6)\left(x^{4}+\right.$ $\left.20 x^{3}-60 x^{2}-80 x+112\right)^{6}$. So the D-spectrum of subdivision of Heawood graph is the union of $\left\{71 \pm \sqrt{4663},-2^{(8)},-6\right\}$ and the set of roots of the polynomial $\left(x^{4}+20 x^{3}-60 x^{2}-80 x+112\right)^{6}$.

Example 3.2. We know that the Petersen graph is the minimal $(3,5)$-cage. By Theorem [3.3, $Q=\left(\begin{array}{ll}30 & 51 \\ 34 & 52\end{array}\right)$ is a quotient matrix of the distance matrix of subdivision of Petersen graph, and its characteristic polynomial is $x^{2}-82 x-174$. So the distance spectral radius of subdivision of Petersen graph is $41+\sqrt{1855}$. We also compute the distance characteristic polynomial of subdivision of Petersen graph, which is $\left(x^{2}-82 x-174\right)\left(x^{2}+16 x-4\right)^{5}\left(x^{2}-\right.$ $2 x-4)^{4}(x+2)^{5}$. So the D-spectrum of subdivision of minimal $(3,5)$-cage is 
$\left\{41 \pm \sqrt{1855},(-8 \pm 2 \sqrt{17})^{(5)},(1 \pm \sqrt{5})^{(4)},-2^{(5)}\right\}$.

In the next two theorems we find distance spectrum of subdivision of minimal $(k, 3)$-cages (complete graphs $\left.K_{k+1}\right)$ and $(k, 4)$-cages (complete bipartite graphs $K_{k, k}$ ). We denote the $m \times n$ all one matrix by $J_{m \times n}$ (or simply by $J$ if its order is clear from the context) and an $n$-dimensional all one vector by $1_{n}$.

Theorem 3.4. The distance spectrum of subdivision of a minimal $(k, 3)$-cage is $\left\{k^{2} \pm \sqrt{\frac{1}{2} k\left(k^{2}+1\right)(2 k+1)},(-2 k)^{(k)}, 0^{\left(\left(_{2}^{k+1}\right)-1\right)}\right\}$.

Proof. The block matrix representation of $D\left(S\left(K_{k+1}\right)\right)$ with respect to the bipartition $V_{1} \cup V_{2}$ of $S\left(K_{k+1}\right)$ is given by

$D\left(S\left(K_{k+1}\right)\right)=\left[\begin{array}{cc}2\left(J_{k+1 \times k+1}-I_{k+1 \times k+1}\right) & 3 J_{k+1 \times\left(\begin{array}{c}k+1 \\ 2\end{array}\right)}-2 R_{k+1 \times\left(\begin{array}{c}k+1 \\ 2\end{array}\right)} \\ 3 J_{\left(\begin{array}{c}k+1 \\ 2\end{array}\right) \times k+1}-2 R_{\left(\begin{array}{c}k+1 \\ 2\end{array}\right) \times k+1}^{T} & 4 J_{\left(\begin{array}{c}k+1 \\ 2\end{array}\right) \times\left(\begin{array}{c}k+1 \\ 2\end{array}\right)-2 R_{\left(\begin{array}{c}k+1 \\ 2\end{array}\right) \times k+1} R_{k+1 \times\left(\begin{array}{c}k+1 \\ 2\end{array}\right)}}\end{array}\right]$,

where $J$ is the all one matrix and $R$ is the incidence matrix of $K_{k+1}$. The adjacency spectrum of $K_{k+1}$ is $\left\{k,-1^{(k)}\right\}$. Let $X$ be an eigenvector of $A\left(K_{k+1}\right)$ corresponding to the eigenvalue -1 . So $X$ is orthogonal to the all one vector. Also by Lemma 1.6, $R R^{T}=A\left(K_{k+1}\right)+k I$. Thus

$$
\begin{gathered}
{\left[\begin{array}{cc}
2(J-I) & 3 J-2 R \\
3 J-2 R^{T} & 4 J-2 R^{T} R
\end{array}\right]\left[\begin{array}{c}
X \\
R^{T} X
\end{array}\right]=\left[\begin{array}{c}
-2 X-2 R R^{T} X \\
-2 R^{T} X-2 R^{T} R R^{T} X
\end{array}\right]} \\
=\left[\begin{array}{c}
-2 X-2(k-1) X \\
-2 R^{T} X-2(k-1) R^{T} X
\end{array}\right]=-2 k\left[\begin{array}{c}
X \\
R^{T} X
\end{array}\right] .
\end{gathered}
$$

So $-2 k$ is an eigenvalue of $D\left(S\left(K_{k+1}\right)\right)$ with multiplicity $k$.

Let $Y$ be an eigenvector of $J_{\left(\begin{array}{c}k+1 \\ 2\end{array}\right) \times\left(\begin{array}{c}k+1 \\ 2\end{array}\right)}$ corresponding to the eigenvalue 0 with multiplicity $\left(\begin{array}{c}k+1 \\ 2\end{array}\right)-1$. Then $Y$ is orthogonal to the all one vector. Now

$$
\left[\begin{array}{cc}
2(J-I) & 3 J-2 R \\
3 J-2 R^{T} & 4 J-2 R^{T} R
\end{array}\right]\left[\begin{array}{c}
R Y \\
-Y
\end{array}\right]=\left[\begin{array}{c}
-2 R Y+2 R Y \\
-2 R^{T} R Y+2 R^{T} R Y
\end{array}\right]=0\left[\begin{array}{c}
R Y \\
-Y
\end{array}\right] .
$$

So 0 is an eigenvalue of $D\left(S\left(K_{k+1}\right)\right)$ with multiplicity $\left(\begin{array}{c}k+1 \\ 2\end{array}\right)-1$. Now the eigenvectors $\left[\begin{array}{c}X \\ R^{T} X\end{array}\right]$ and $\left[\begin{array}{c}R Y \\ -Y\end{array}\right]$ of $D\left(S\left(K_{k+1}\right)\right)$ are orthogonal to $\left[\begin{array}{c}1_{k+1} \\ 0\end{array}\right]$ and $\left[\begin{array}{c}0 \\ 1\left(\begin{array}{c}k+1 \\ 2\end{array}\right)\end{array}\right]$ respectively. So every other eigenvector $Z$ of $D\left(S\left(K_{k+1}\right)\right)$ is of the 
form $\left[\begin{array}{c}a 1_{k+1} \\ b 1_{\left(\begin{array}{c}k+1 \\ 2\end{array}\right)}\end{array}\right], a, b \neq 0$. Now $D\left(S\left(K_{k+1}\right)\right) Z=\lambda Z$ implies,

$$
\begin{aligned}
& 2 k a+\frac{1}{2} k(3 k-1) b=\lambda a, \\
& (3 k-1) a+2 k(k-1) b=\lambda b
\end{aligned}
$$

Since $a, b \neq 0$, solving the above equations we get $\lambda^{2}-2 k^{2} \lambda-\frac{1}{2} k(k+1)^{2}=0$, and hence the result.

Theorem 3.5. The distance spectrum of subdivision of a minimal $(k, 4)$-cage is $\left\{2 k^{2}+k-2 \pm \sqrt{\left(4 k^{4}-2 k^{3}+9 k^{2}-12 k+4\right)}, 2 k-4,0^{\left((k-1)^{2}\right)},(-(k+2)+\right.$ $\left.\left.\sqrt{k^{2}+4}\right)^{(2 k-2)},\left(-(k+2)-\sqrt{k^{2}+4}\right)^{(2 k-2)}\right\}$.

Proof. We take the vertex partition of $S\left(K_{k, k}\right)$ as $V_{1} \cup V_{2} \cup V_{3}$, where $\left(V_{1}, V_{2}\right)$ is bipartition of $K_{k, k}$ and $V_{3}$ is the set of all new vertices inserted on edges of $K_{k, k}$. The distance matrix of $S\left(K_{k, k}\right)$ can be written as, $D\left(S\left(K_{k, k}\right)\right)=$ $\left[\begin{array}{ccc}4\left(J_{k \times k}-I_{k \times k}\right) & 2 J_{k \times k} & \left(3 J_{k \times k}-2 I_{k \times k}\right) \otimes 1_{k}^{T} \\ 2 J_{k \times k} & 4\left(J_{k \times k}-I_{k \times k}\right) & 1_{k}^{T} \otimes\left(3 J_{k \times k}-2 I_{k \times k}\right) \\ \left(3 J_{k \times k}-2 I_{k \times k}\right) \otimes 1_{k} & 1_{k} \otimes\left(3 J_{k \times k}-2 I_{k \times k}\right) & 4\left(J_{k^{2} \times k^{2}}-I_{k^{2} \times k^{2}}\right)-2 A\left(L\left(K_{k, k}\right)\right)_{k^{2} \times k^{2}}\end{array}\right]$. Adjacency matrix $A\left(K_{k, k}\right)$ and incidence matrix $R$ of $K_{k, k}$ are $\left[\begin{array}{cc}0 & J_{k \times k} \\ J_{k \times k} & 0\end{array}\right]$ and $\left[\begin{array}{c}I_{k \times k} \otimes 1_{k}^{T} \\ 1_{k}^{T} \otimes I_{k \times k}\end{array}\right]$ respectively. Thus $3 J_{k \times k^{2}}-2 R_{k \times k^{2}}=\left[\begin{array}{c}\left(3 J_{k \times k}-2 I_{k \times k}\right) \otimes 1_{k}^{T} \\ 1_{k}^{T} \otimes\left(3 J_{k \times k}-2 I_{k \times k}\right)\end{array}\right]$. Let $X$ be an eigenvector of $A\left(L\left(K_{k, k}\right)\right)$ corresponding to the eigenvalue -2 with multiplicity $(k-1)^{2}$. Applying Lemma 1.7 we have $R X=0$. So $X$ is orthogonal to the all one vector. Now

$$
\begin{gathered}
{\left[\begin{array}{ccc}
4(J-I) & 2 J & (3 J-2 I) \otimes 1_{k}^{T} \\
2 J & 4(J-I) & 1_{k}^{T} \otimes(3 J-2 I) \\
(3 J-2 I) \otimes 1_{k} & 1_{k} \otimes(3 J-2 I) & 4(J-I)-2 A\left(L\left(K_{k, k}\right)\right)
\end{array}\right]\left[\begin{array}{c}
0 \\
0 \\
X
\end{array}\right]} \\
=\left[\begin{array}{c}
\left((3 J-2 I) \otimes 1_{k}^{T}\right) X \\
\left(1_{k}^{T} \otimes(3 J-2 I)\right) X \\
-4 X+4 X
\end{array}\right]=\left[\begin{array}{c}
(3 J-2 R) X \\
0
\end{array}\right]=0\left[\begin{array}{c}
0 \\
0 \\
X
\end{array}\right] .
\end{gathered}
$$

Thus 0 is an eigenvalue of $D\left(S\left(K_{k, k}\right)\right)$ with multiplicity $(k-1)^{2}$.

Let $Z$ be an eigenvector of $A\left(K_{k, k}\right)$ corresponding to the eigenvalue 0 with multiplicity $2 k-2$. Also let $X^{\prime}$ and $Y$ be vectors orthogonal to the all one vector $1_{k}$. If $\left[\begin{array}{c}X^{\prime} \\ Y \\ Z\end{array}\right]$ happens to be an eigenvector of $D\left(S\left(K_{k, k}\right)\right)$ corresponding 
to an eigenvalue $\lambda$, then it must satisfy,

$$
\left[\begin{array}{ccc}
4(J-I) & 2 J & (3 J-2 I) \otimes 1_{k}^{T} \\
2 J & 4(J-I) & 1_{k}^{T} \otimes(3 J-2 I) \\
(3 J-2 I) \otimes 1_{k} & 1_{k} \otimes(3 J-2 I) & 4 J-2 R^{T} R
\end{array}\right]\left[\begin{array}{c}
X^{\prime} \\
Y \\
Z
\end{array}\right]=\lambda\left[\begin{array}{c}
X^{\prime} \\
Y \\
Z
\end{array}\right]
$$

This implies,

$$
\begin{aligned}
& -4 X^{\prime}+\left((3 J-2 I) \otimes 1_{k}^{T}\right) Z=\lambda X^{\prime} \\
& -4 Y+\left(1_{k}^{T} \otimes(3 J-2 I)\right) Z=\lambda Y \\
& \left\{(3 J-2 I) \otimes 1_{k}\right\} X^{\prime}+\left\{1_{k} \otimes(3 J-2 I)\right\} Y-2 R^{T} R Z=\lambda Z .
\end{aligned}
$$

Let $W=\left[\begin{array}{c}X^{\prime} \\ Y\end{array}\right]$. Combining the relations we get,

$$
\begin{aligned}
& -4 W+(3 J-2 R) Z=\lambda Z, \\
& \left(3 J^{T}-2 R^{T}\right) W-2 R^{T} R Z=\lambda Z .
\end{aligned}
$$

From the first equation we get, $-4 W-2 R Z=\lambda Z, R Z=-\frac{1}{2}(\lambda+4) W$. Applying Lemma [1.6 in the second equation we get

$$
\begin{aligned}
& -2 R R^{T} W-2 R R^{T} R Z=\lambda R Z,-2(A+k I) W-2(A+k I) R Z=\lambda R Z, \\
& -2 k W+k(\lambda+4) W=-\frac{1}{2} \lambda(\lambda+4) W .
\end{aligned}
$$

Thus $\lambda^{2}+(2 k+4) \lambda+4 k=0$. So $-(k+2) \pm \sqrt{k^{2}+4}$ are two eigenvalues of $D\left(S\left(K_{k, k}\right)\right)$ with multiplicity $2 k-2$. Now $\left[\begin{array}{c}0 \\ 0 \\ X\end{array}\right]$ is orthogonal to $\left[\begin{array}{c}0 \\ 0 \\ 1_{k^{2}}\end{array}\right]$ and $\left[\begin{array}{c}X^{\prime} \\ Y \\ Z\end{array}\right]$ is orthogonal to both $\left[\begin{array}{c}1_{k} \\ 0 \\ 0\end{array}\right]$ and $\left[\begin{array}{c}0 \\ 1_{k} \\ 0\end{array}\right]$. So every other eigenvector $U$ of $D\left(S\left(K_{k, k}\right)\right)$ is of the form $\left[\begin{array}{c}a 1_{k} \\ b 1_{k} \\ c 1_{k^{2}}\end{array}\right], a, b, c \neq 0$. Now $D\left(S\left(K_{k \times k}\right)\right) U=\mu U$ implies,

$$
\begin{aligned}
& 4(k-1) a+2 k b+k(3 k-2) c=\mu a \\
& 2 k a+4(k-1) b+k(3 k-2) c=\mu b \\
& (3 k-2) a+(3 k-2) b+4 k(k-1) c=\mu c .
\end{aligned}
$$

Since $a, b, c \neq 0$, solving the above equations we get $\mu^{3}-\left(4 k^{2}+4 k-8\right) \mu^{2}+$ $\left(14 k^{3}-28 k^{2}-8 k+16\right) \mu-\left(12 k^{4}-56 k^{3}+80 k^{2}-32 k\right)=0$. So $(\mu-2 k+$ $4)\left(\mu^{2}-\left(4 k^{2}+2 k-4\right) \mu+\left(6 k^{3}-16 k^{2}+8 k\right)=0\right.$, and hence the result. 
Remark 3.1. Theorem 3.2 also gives that the distance spectral radius of subdivision of minimal $(k, 4)$-cage is $2 k^{2}+k-2+\sqrt{\left(4 k^{4}-2 k^{3}+9 k^{2}-12 k+4\right)}$.

\section{Concluding Remarks}

It is known that a distance regular graph of diameter $d$ has exactly $d+1$ distinct eigenvalues. However this is not the case for distance eigenvalues. The authors in [5] proved that every distance regular graph of diameter $d$ has at the most $d+1$ distinct distance eigenvalues and asked for characterization of distance regular graphs which have exactly $d+1$ distinct distance eigenvalues. In this paper we proved that all minimal cages have exactly $d+1$ distinct distance eigenvalues. We also found distance spectral radius of DBR graphs and determined the full distance spectrum for some DBR graphs associated with minimal $(k, g)$-cages. For the remaining, the following matrix representation of distance matrix of subdivision of a minimal $(k, g)$-cage $G$ may be useful. Here we consider $\left(V_{1}, V_{2}\right)$ as the vertex partition of subdivision of a minimal $(k, g)$-cage $G$, where $V_{1}=V(G)$ and $V_{2}$ is the set of all new vertices inserted on edges of $G$.

So for $g$ even, $D(S(G))=\left[\begin{array}{cc}2 D(G) & \frac{1}{2} D(G) R(G) \\ \frac{1}{2} R(G)^{T} D(G)^{T} & 2 D(L(G)) \\ \text {. } & \end{array}\right]$, and for $g$ odd, $D(S(G))=\left[\begin{array}{cc}2 D(G) & \frac{1}{2} D(G) R(G)+E \\ \frac{1}{2} R(G)^{T} D(G)^{T}+E^{T} & 2 D(L(G)) \\ . & \end{array}\right]$, where $R(G), D(G)$ and $D(L(G))$ are incidence matrix, distance matrix and distance matrix of the line graph of $G$ respectively. $E$ is a matrix whose rows are indexed by vertices of $G$ and columns are indexed by vertices on $V_{2}$ and $(i, j)^{t h}$ entry of $E$ is 1 if $d\left(v_{i}, u_{j}\right)=\max \left\{d(v, u): v \in V_{1}, u \in V_{2}\right\}$ and 0 otherwise.

\section{Acknowledgement}

The first author is grateful to Council of Scientific and Industrial Research (CSIR), India [Grant number: 09/081(1283)/2016-EMR - I], for funding the research. 


\section{References}

[1] A. Alazemi et al. "Distance-regular graphs with small number of distinct distance eigenvalues." Linear Algebra and its Applications 531 (2017): 83-97.

[2] G. Aalipour et al. "On the distance spectra of graphs." Linear Algebra and its Applications 497 (2016): 66-87.

[3] M. Aouchiche, and P. Hansen. "Distance spectra of graphs: A survey." Linear algebra and its applications 458 (2014): 301-386.

[4] F. Atik, and P. Panigrahi, "Distance Spectral Radius of Some kpartitioned Transmission Regular Graphs." Conference on Algorithms and Discrete Applied Mathematics. Springer, Cham, 2016.

[5] F. Atik, and P. Panigrahi, "On the distance spectrum of distance regular graphs." Linear Algebra and its Applications 478 (2015): 256-273.

[6] N. Biggs, N. L. Biggs, and B. Norman, "Algebraic graph theory." Vol. 67. Cambridge university press, 1993.

[7] A. E. Brouwer,W.H. Haemers, Spectra of Graphs. Springer, New York (2011).

[8] A. E. Brouwer, A. M. Cohen, and A. Neumaier. "Distance-Regular Graphs." (1989).

[9] K. Balasubramanian, "Computer generation of distance polynomials of graphs." Journal of Computational Chemistry 11.7 (1990): 829-836.

[10] D. Cvetkovic, M. Doob, and H. Sachs. "Spectra of Graphs-Theory and Application. 1980." Pure and Applied Mathematics (1980).

[11] G. Exoo, and R. Jajcay, "Dynamic cage survey." The electronic journal of combinatorics (2012): DS16-July.

[12] C.D. Godsil, and J. Shawe-Taylor. "Distance-regularised graphs are distance-regular or distance-biregular." Journal of Combinatorial Theory, Series B 43.1 (1987): 14-24.

[13] R.L. Graham, and H.O. Pollak, "On the addressing problem for loop switching." The Bell System Technical Journal 50.8 (1971): 2495-2519.

[14] A. Graovac, G. Jashari, and M. Strunje. "On the distance spectrum of a cycle." Aplikace matematiky 30.4 (1985): 286-290. 
[15] H. Lin, et al. "A survey on distance spectra of graphs." Adv. Math.(China) 50.01 (2021): 29-76.

[16] B. Mohar and J. Shawe-Taylor, "Distance-biregular graphs with 2-valent vertices and distance-regular line graphs." Journal of Combinatorial Theory, Series B 38.3 (1985): 193-203.

[17] K. Schacke, "On the kronecker product", Master's thesis, University of Waterloo (2004).

[18] D. Stevanović, and G. Indulal. "The distance spectrum and energy of the compositions of regular graphs." Applied mathematics letters 22.7 (2009): 1136-1140.

[19] ER. van Dam, JH. Koolen, and H. Tanaka, "Distance-Regular Graphs." The Electronic Journal of Combinatorics: EJC (2016): 1-156. 